\title{
MedienPädagogik
}

Zeitschrift für Theorie und Praxis der Medienbildung

Jahrbuch Medienpädagogik 4.

Zweitveröffentlichung aus: Jahrbuch Medienpädagogik 4. (2005) Wiesbaden: VS Verlag für Sozialwissenschaften. Hrsg. v. Ben Bachmair, Peter Diepold und Claudia de Witt.

\section{Medienkompetenz als theoretisches Konzept und Gegenstand empirischer Forschung}

\author{
Dorothee M. Meister, Jörg Hagedorn und Uwe Sander
}

\section{Einführung}

Der Beitrag versteht sich als Teil der Bemühung, das Phänomen Medienkompetenz bei Jugendlichen besser beschreiben und analysieren zu können. Dabei gehen wir von einem Verständnis von Medienkompetenz aus, das sich an den von Dieter Baacke (1996) vorgeschlagenen vier Dimensionen (Medienkritik, Mediennutzung, Medienkunde, Mediengestaltung) orientiert. Im Rahmen des DFG-Projektes „Untersuchung zum Mediennutzungsverhalten 13-18jähriger und Entwicklung von Medienkompetenz im Jugendalter“1 untersuchen wir empirisch das Medienhandeln Jugendlicher in Anlehnung an das Bielefelder Medienkompetenzmodell (Treumann/Baacke u.a. 2002). In einer quantitativen Befragung haben wir zunächst die Dimensionen der Medienkompetenz operationalisiert und erhoben. Des Weiteren konkretisieren wir in qualitativen Befragungen die Medienkompetenz bei Jugendlichen inhaltlich und erschließen diese in ausgewählten Fällen auch rekonstruktiv hermeneutisch. Die Einzelinterviews dienen dazu, das Medienhandeln und die verschiedenen Ebenen der Medienkompetenz umfassend zu erheben und im Rahmen einer Clusteranalyse mit den quantitativen Daten in Beziehung zu setzen. Im Rahmen von Gruppendiskussionen werden indes die kollektiven Orientierungen Jugendlicher in der analytisch-reflexiven Auseinandersetzung mit Medien und damit die Dimension der Medienkritik empirisch erschlossen.

In diesem Beitrag stellen wir zwei Zugänge bei der Auswertung der Gruppendiskussionen vor. Beim ersten Zugang - hier orientieren wir uns stark an dem Medienkompetenzmodell von D. Baacke - werden die konkreten Sichtweisen und Orientierungen der Jugendlichen in einer kritischen Auseinandersetzung mit medial vermittelten Ereignissen herausgearbeitet. Dieser Zugang geht der Frage nach, welche analytisch-reflexiven Fähigkeiten sich in der Ausprägung von Medienkritik in den Aussagen der Jugendlichen aufspüren lassen. Beim zweiten Zugang nähern wir uns den Aussagen der Jugendlichen

1 Das Projekt läuft seit Januar 2001 an den Standorten Bielefeld, Rostock und Halle. Leitung: Prof. Dr. Klaus Treumann, Prof. Dr. Uwe Sander, Dr. Dorothee M. Meister. Projektmitarbeit: Eckard Burkatzki, Jörg Hagedorn, Mareike Strotmann, Dr. Claudia Wegener 
in einer rekonstruktiv hermeneutischen Logik. Mittels einer exemplarischen Fallrekonstruktion verdeutlichen wir, wie kollektiv generierte Orientierungsrahmungen in der Auseinandersetzung mit alltagsrelevanten Handlungsproblemen ausschlaggebend dafür sind, wie Jugendliche zu einer spezifischen Ausprägung von Medienkompetenz gelangen können. Medienkritik wird in diesem Fall von der kollektiven Interpretation gesellschaftlicher Modernisierungs- und Wandlungsprozesse bestimmt. Insbesondere sind es die milieu- bzw. bildungsspezifischen Dispositionen Jugendlicher, die in der Generierung konkreter Orientierungsrahmungen festschreiben, zu welchen Formen der Ausprägungen von Medienkritik Jugendliche in der Lage sind und welche sie für sich selbst ausschließen.

\section{Zur theoretischen Konzeption von Medienkompetenz}

Auch wenn der Begriff der Medienkompetenz vielfach Verwendung findet, so ist seine theoretische Verankerung in den Sozialwissenschaften kaum kanonisiert, wodurch die Definitionsvielfalt teilweise erklärt werden kann. Ein Ansatz, das Konzept theoretisch zu, stammt von Dieter Baacke, der den Begriff Medienkompetenz zum einen aus älteren sozialwissenschaftlichen Debatten zur ,kommunikativen Kompetenz' herleitet und zum anderen auf die Anschlussfähigkeit des Konzeptes zu aktuellen medientheoretischen Debatten verweist wie der Wissensklufthypothese, dem Nutzenansatz, dem Habituskonzept oder dem sozialökologischen Ansatz (vgl. Treumann/Baacke u.a. 2002, S. 19ff.).

In den Überlegungen von Baacke wird Medienkompetenz als eine besondere Form von kommunikativer Kompetenz (gemeint sind dabei alle Sinnesakte der Wahrnehmung) und Handlungskompetenz (gemeint ist damit eine Spezifik von ,Weltermächtigung‘ und handelnder ,Weltveränderung') betrachtet. Als anthropologische Grundkonstante ist dieser Überlegung inhärent, dass der Mensch generell als ein kompetentes Wesen angesehen wird. Kompetenz wird in diesem Kontext nicht wie in der Umgangssprache als quasi expertenhafte Zuständigkeit und Fähigkeit verwendet. Auch geht es bei diesen Annahmen weniger um die Intention der allgemeinen Erziehungswissenschaft, die unter Kompetenz die generative Fähigkeit von Menschen versteht, Wissens- und Handlungsmuster situationsadäquat entwickeln und anwenden zu können (vgl. Baacke u.a. 1999, S. 52f.). Vielmehr rekurriert Baacke auf den Ursprung des Kompetenzbegriffs, die Biologie, die unter Kompetenz (auch: Zuständigkeit) zunächst die (zeitlich begrenzte) Bereitschaft embryonaler Zellen bezeichnet, auf einen bestimmten Entwicklungsreiz zu reagieren. Übertragen auf Sprache und Sprecher bezeichnet Kompetenz dann „die Fähigkeit des letzteren, über die Sprachrichtigkeit von Sätzen zu entscheiden und eine potentiell unbegrenzte Zahl von Sätzen und Aussagen zu produzieren. Kompetenz meint damit (...) eine in der Sprache ange- 
legte Verfügung über den Sinn und die Intention von Aussagen“ (Baacke 1980, S. 261). Damit orientiert sich Baacke am Kompetenzbegriff, wie ihn der Linguisten Chomsky annahm, nämlich als eine im Mentalen verankerte Fähigkeit des Menschen, aufgrund eines immanenten Regelsystems eine potenziell unbegrenzte Anzahl von Sätzen zu erzeugen. Die Vorstellung einer „universellen Grammatik“ behauptet im Grunde, „dass alle Menschen potientiell über die Sprachmuster einer Universalsprache verfügen - und insofern gleich sind“ (Baacke 1996, S. 116). Die tatsächliche Erzeugung eines konkreten Satzes belegt dieses Modell mit dem Begriff der Performanz belegt. Eine empirische Beobachtung vorhandener Kompetenz, genauso wie der Ansatzpunkt pädagogischer Interventionen, kann insofern lediglich auf der Performanzebene erfolgen. Die Herausforderung für die Pädagogik besteht somit im Spannungsverhältnis zwischen Kompetenz und Performanz und damit in der ,Nichtidentität‘ eines universalen Regelsystems und regelgeleiteter aktueller Strukturierung in einer konkreten Sprechsituation. Baacke zieht aus dieser Differenz von Kompetenz und Performanz den Schluss, dass der ontologische Kompetenzbegriff auf ein Gleichheitspostulat hinausläuft, das in diesem Kontext auch pädagogische Förderung begründet, um die allen inhärente Kompetenz zur Performanz zu bringen. Theoretisch begründbar wird eine pädagogische Relevanz zudem aus dem Umstand, dass die Performanzebene als empirisch wahrnehmbare Oberflächenstruktur menschlichen Handelns durch weitere Bedingungen wie subjektive Faktoren (Lebensgeschichte, Motivation) und gesellschaftliche Variablen (institutionelle Bedingungen des Handelns, Rollenerwartungen an den Handelnden) sowie durch situative, entwicklungsbedingte, soziale und kulturelle Variablen beeinflusst wird. Pädagogische Vermittlungsprozesse zielen insofern auf die Transformationsprozesse, mittels derer sich Kompetenz in Performanz realisiert. Insofern ,geht es in impliziten Bildungsprozessen nicht um den Erwerb konkreter Handlungsmuster, sondern um den Erwerb von Strukturen“ (Dewe/ Sander 1996, S. 129), da Kompetenz als kognitive Fähigkeit keine sachliche Phänomenebene, sondern die „Fähigkeit im Umgang mit Wissen selbst“ (ebd., S. 128) betrifft.

Baacke (1997; 1996) entwickelt das Konzept der ,Medienkompetenz ' im Kontext kommunikativer Kompetenz. Er leitet diesen Terminus des Weiteren aus einem „kulturellen In-der-Welt-Sein“ und einem gemeinsamen Wahrnehmungsbewältigungsprozess ab. Betont wird dabei die Tatsache, dass alle Menschen grundsätzlich mit der Fähigkeit ausgestattet sind, sich in der Welt erfolgreich und sozial zu bewegen, allerdings muss diese Ausstattung gefördert, zur Performanz gebracht werden. Zur Operationalisierung des Konzeptes schlägt Baacke vor, vier Dimensionen von Medienkompetenz zu unterscheiden, die jeweils aus mehreren Unterdimensionen bestehen: Medienkritik, Medienkunde, Mediennutzung und Mediengestaltung (vgl. dazu ausführlicher Treumann u.a. in diesem Band). Medienkompetenz „meint also grundlegend nichts anderes als die Fähigkeit, in die Welt aktiv aneignender Weise auch alle Arten von Medien für das Kommunikations- und Handlungsrepertoire von Menschen einzusetzen.“ (Baacke 1996, S. 119). 
Medienkompetenz stellt insofern einen Sammelbegriff dar mit allgemeinen Anforderungen, die Kompetenz- und Performanzelemente enthalten, was in modernen Gesellschaften bedeutet, allgemeine Kriterien mit spezifischen Erwartungen zu verbinden. Doch die Konkretion dessen, welche spezifischen Kenntnisse und Eigenschaften eine medienkompetente Person vereinen sollte, lässt sich nicht aus der Theorie allein beziehen, sondern bedarf einer aus dem Fall bezogenen empirischen Überprüfung. Das bedeutet auch, es bedarf noch genauerer Untersuchungen, wie Medienkompetenzen in den Alltag eingebunden sind und welche Erscheinungsformen und Ausprägungen wir vorfinden. ${ }^{2}$

Qualitative Verfahren können nun genau hier ansetzen. Gerade der Bereich der Medienkritik ${ }^{3}$ ist in diesem Zusammenhang von besonderem Interesse, da diese von Pädagogen häufig eingeklagt wird, es aber nach wie vor unklar ist, wie sich eine reflexivanalytische Medienkompetenz im Alltag zeigt. Hierzu sind Studien, die die hermeneutische Rekonstruktion jugendlicher (kollektiver) Sinn- und Bedeutungszuschreibungen in Bezug auf das eigene Medienverhalten zum Gegenstand ihrer Forschung machen, bisher nahezu gänzlich ausgeblieben. So bleibt es bislang offenbar unklar, inwiefern die ambivalente Dynamik zwischen sozialräumlichen Bedingungen und individuellen Prozessen noch genauer gefasst werden kann (vgl. Thole 2002; S. 678). Darüber hinaus wird zwar immer wieder gerade in den sozialisationstheoretischen bzw. alltags- und lebensweltlichen Ansätzen der Medienforschung auf die (selbst-)sozialisatorische Potenzialität hingewiesen, die jugendliche Peers in der Auseinandersetzung mit Medien freisetzen können (vgl. Thole 2002; Barthelmes/Sander 1997; Loos/Schäffer 2001; Fromme u.a. 1999). Auch hierzu liegen der Medienforschung kaum fundierte, erkenntnisgenerierende Studien vor. So bleibt eine Lücke in der Medienforschung gerade an der Stelle festzustellen, wo es um die methodisch fundierte Herleitung jugendlicher Sichtweisen und Deutungen eigenen Medienhandelns einerseits und medialen Wirkmechanismen andererseits geht.

Diesem Forschungsdesiderat begegnen wir in diesem Beitrag mittels eines methodisch rekonstruktiven Zugangs in der empirischen Untersuchung von Medienkompetenz, der verdeutlicht, welche spezifisch ausgeformten und rekonstruktiv erschlossenen Sichtweisen und Deutungen Jugendliche ihrer ,Interpretation` medienkritischen Handelns zur Seite stellen.

2 Wie wir das Thema Medienkompetenz mittels quantitativer Fragen untersuchen, führt der Beitrag von Treumann u.a. in diesem Band aus. Insbesondere in Bezug auf die Dimension der Medienkritik hat sich in der Praxis die Operationalisierung von Medienkritik als besonders schwierig erwiesen.

3 Medienkritik umfasst nach Baacke eine analytische, ethische und reflexive Dimension. 


\section{Empirische Studie zur Erforschung von Medienkompetenz bei Jugendlichen}

In der quantitativen Befragung konnten wir die Medienkritik Jugendlicher nur eingeschränkt erheben bzw. erschließen. Um nun genauere Aussagen zur Medienkritik Jugendlicher treffen zu können, haben wir in unserem Untersuchungssetting das Gruppendiskussionsverfahren übernommen. Die Wahl dieses Verfahrens war zweifach motiviert: Einerseits war es uns wichtig, eine große Anzahl von Meinungen Jugendlicher vorzulegen, um so das breite Spektrum an medienkritischen Haltungen von Jugendlichen zu erfassen. Andererseits besteht mit Gruppendiskussionen die Möglichkeit, kollektive Deutungen aufzudecken, die spezifische Haltungen und performative Erscheinungen erklären (vgl. Loos/Schäffer 2001). In drei Bundesländern wurden insgesamt zehn Gruppendiskussionen durchgeführt mit Gruppen von Schülerinnen und Schülern aus verschiedenen Schultypen. Die Auswertung der qualitativen, transkribierten Gruppendiskussionen erfolgte in mehreren Schritten. Zunächst wurde der Text nach Textpassagen und nach bestimmten Codes geordnet. Diese Codebäume wurden mit Hilfe eines Softwareprogramms (MaxQda) erstellt. Die Auswertung der Datenmaterialien erfolgte zunächst nach den thematischen (Medienkritik-) Dimensionen, die sich aus dem Thema der Gruppendiskussion ergaben (Bohnsack 1997). In einem zweiten, exemplarisch durchgeführten Schritt wurden einzelne Textpassagen hermeneutisch rekonstruiert, um so kollektive Deutungen zu ermitteln (Oevermann 2001, 2001a).

Grundlage der folgenden Überlegungen ist eine Gruppendiskussion, die mit einer Berufsschulklasse in einer Mittelstadt in Sachsen-Anhalt im Frühjahr 2002 geführt wurde. Teilgenommen haben an dem ca. zwei Stunden dauernden Gespräch drei Jungen und vier Mädchen einer Restaurantfachklasse.

\subsection{Aufbau und Durchführung der Gruppendiskussion zur Erhebung von Medienkritik} Als Aufhänger für die Gruppendiskussionen wurde die Medienberichterstattung rund um den „11. September“ (Anschlag auf das WTC im Jahre 2001) gewählt. Wie auch bei den anderen Gruppendiskussionen stand bei der hier thematisierten Gruppe von Berufsschülern aus einer Mittelstadt in Sachsen- Anhalt der Fokus des Interesses auf den reflexiven und analytischen Fähigkeiten der Jugendlichen in Bezug auf Medien (Medienkritik, eingebunden in das Konzept von Medienkompetenz).

Als Stimulus zeigten wir den Jugendlichen zunächst vier Fotos des Ereignisses. Ziel dieses neuen Verfahrens in der Gruppendiskussion (vgl. Loos/Schäffer 2001) war, über die Bilder das Vergangene zu vergegenwärtigen. 
Die Diskussion begann mit der Frage, wie die Jugendlichen von dem Ereignis erfuhren. Danach brachten die Moderatoren auch Leitfragen zu analytischen, reflexiven und ethischen Bezügen von Medienkritik ein. So wurde beispielsweise im Hinblick auf eine analytische Dimension gefragt, ob wir uns sicher sein können, dass die Berichterstattung wirklich das zeigt, was vor Ort passiert ist. Hinsichtlich ethischer Überlegungen legten wir den Jugendlichen eine weitere Serie von Fotos über die Ereignisse rund um den „11. September“ vor. So versetzten wir die Jugendlichen quasi in die Rolle von Medienproduzenten und baten sie zu begründen, welche Bilder sie für eine Veröffentlichung eines Artikels in einer Zeitung auswählen würden. In der Logik eines ersten Zuganges zu dieser Gruppendiskussion haben wir erwartet, dass sich aus den Sichtweisen der Jugendlichen in der Auseinandersetzung mit den Ereignissen des 11. September ein starkes Informationsbedürfnis zeigen würde, die Jugendlichen Reflexionen zu dem Ereignis selbst anstellen und sie eine differenzierte Reflexion der Rolle der Medien als auch eine Bewertung der Medien vornehmen würden.

In einem ersten Schritt zeichnen wir nach, wie die Jugendlichen diese Ereignisse in ihrer medialen Vermittlung erfahren haben, welche je spezifischen Strategien sie in der Verarbeitung dieser Ereignissen entwickelt haben und welche - auch alltagsrelevanten - Erwartungsansprüche sie vor dem Hintergrund ihrer kollektiven Medienerfahrungen an die Medien selbst adressieren. In einem zweiten Schritt werden wir die sinn- und bedeutungsstiftenden kollektiven Orientierungsrahmungen rekonstruktiv herleiten, die als Begründungsfolie für das eigene Medienhandeln, aber auch für die konkret ausgeformten und im ersten Schritt nachgezeichneten Erwartungshaltungen dienen, die von den Jugendlichen an die Medien adressiert werden.

\subsection{Medienkritische Haltungen einer Berufsschulklasse}

Das Ereignis des 11. September veranschaulicht, wie stark die Mediennutzung der Jugendlichen lebensweltlich eingebettet ist. So durchbrechen die Ereignisse dieses Tages die Alltags- und Handlungsgewohnheiten der Jugendlichen und führen zu erheblichen Irritationen. Zur Wiederherstellung von ,Weltsicherheit' streben die Berufsschüler allerdings keine komplexe Aufarbeitung von Hintergründen an, sondern sind daran interessiert, über die Medien eindeutige Konsequenzen vermittelt zu bekommen und damit Orientierungen zu erhalten, die eine weitere Auseinandersetzung und Information über ,Hintergründe“ nicht mehr erforderlich machen.

Der Verlauf der Gruppendiskussion zeigt, dass die Jugendlichen von dem Anschlag relativ schnell erfahren haben; jedoch konnten sie aus den Berichterstattungen kaum verlässliche und eindeutige Informationen beziehen. So haben die Jugendlichen über weite Strecken eine ,objektive Medienberichterstattung' vermisst, die es ihnen erleichtert hätte, Weltsicherheit in den medial vermittelten Ereignissen über eine ,richtige‘ Deutung wiederherstellen zu können. 
Vor dem Hintergrund dieser Medienerfahrung, die geprägt war vom Verlust von eindeutigen Wahrheits- und Wirklichkeitskonstruktionen, generierte sich bei den Jugendlichen das Gefühl von Angst und Unsicherheit. Schnell werden die Folgen dieser Ereignisse auch für das eigene Land und für die eigene Sicherheit gedacht. Ängste, die sich um potenzielle globale Kriegsszenarien rankten, wurden nicht zuletzt auch von den Medien selbst geschürt, denn „viele Sender haben geredet, jetzt bricht der Krieg aus“ (Em; Z. 4546). Dieser Krieg hätte dann nicht nur die Konsequenz um das eigene Leben fürchten zu müssen, vielmehr stand für sie die Weltsicherheit an sich in Gefahr: „und dass, wirklich, die Welt nicht mehr lange lebt“ (Em; Z. 487). Dass sich diese Ängste und Unsicherheiten nicht nur an die gegenwärtigen weltpolitischen Krisenkonstellationen - ausgelöst durch die Ereignisse vom „11. September“ - rankten, sondern vielmehr auch in der Zukunft potenziell auf Dauer gestellt sein können, zeigt die Aussage: „man weiß nicht, was in der Zukunft steckt, was da noch alles passieren kann, kann noch viel passieren“ (Em; Z. 821-824). Damit wird die so dramatisch erlebte und nachvollzogene Krisenhaftigkeit in eine potenziell krisenhafte Zukunft überführt. In den Deutungen der Jugendlichen müssen Medien aber die Funktion übernehmen, über eindeutig vermittelte Wahrheiten und Informationen ,Weltsicherheiten` beim Publikum herzustellen. Genau dies gelingt ihnen aber in den Augen der Jugendlichen in diesem Fall nicht.

Diesem Handlungsproblem, sich nicht auf medial vermittelte Weltsicherheiten verlassen zu können, versuchten die Jugendlichen durch einen Rückzug in gewohnte Alltags- und Handlungsroutinen (Schauen von Spielfilmen und Video) zu entkommen. Nachdem die Berichterstattung über die Ereignisse des 11. Septembers bei den Jugendlichen eine gewisse Übersättigung ausgelöst hatten, konnte der Rückzug in habitualisierte Fernsehgewohnheiten freilich nur zum Teil gelingen, was nicht zuletzt daran lag, dass „die ganzen Spielfilme, alle ausgefallen“ (Bw; Z. 218) sind. Insofern waren die Jugendlichen weiterhin der Dramatik der Ereignisse (,diese Bilder andauernd“ (Dm; Z. 291-292)) ausgeliefert, was letztendlich dazu führte, dass sie diese Bilder „zum Schluss gar nicht mehr sehen" (Dm; Z. 292-293) konnten. Auch der antizipierte, entlastende Rückzug in die Sozialbeziehungen (Peers, Elternhaus etc.) war durchzogen von permanenter Konfrontation mit den Ereignissen, kaum gab es darüber hinaus Gespräche mit Freunden und Eltern über diese Ereignisse. Diese Konfrontation im Zusammenhang mit der tendenziell ausgebliebenen Möglichkeit der Aufklärung und Information, ließen die Ereignisse zu einer Art symbolischer Gewalt („,die haben die Leute bloß weiter fertig gemacht“ (Dm; Z. 247-248)) werden (vgl. Bourdieu 1998; S. 21 ff.), der die Jugendlichen individuell und kollektiv allenfalls in Form der Abwehr und Verweigerung entkommen konnten.

Augenscheinlich wird damit der Medienkritik durch diese Jugendlichen eine Absage erteilt. Denn so lassen sich in den Aussagen der Jugendlichen kaum analytische, reflexive und ethische Bezüge in der Deutung und Interpretation dieser Ereignisse nachzeichnen. Vielmehr wird diese Leistung den 
Medien selbst überantwortet. Medien sollten gewissermaßen ihr eigenes Handeln (objektive Berichterstattung, Auswahl der Bilder nach ethischen Dispositionen etc.) selbst reflektieren und in zweifelsfreien Sinn- und Deutungskonstruktionen an die Medienrezipienten vermitteln, also ein ,wahres` Abbild sozialer Wirklichkeit liefern. Eine Leistung bzw. ein Professionalitätsanspruch, den diese Jugendlichen selbst nicht einlösen wollen/ können. ,Wahrheit‘, ,Sinnhaftigkeit‘ und ,Wirklichkeit‘ werden von diesen Jugendlichen also im entscheidenden Maße über die Symbolvorräte der Medien bezogen. Kommen Medien der Vermittlung dieser ,deutungsresistenten` Symbole nicht mehr nach, fehlt es den Jugendlichen an einer stellvertretenden Deutungsinstanz und die Welt wird immer weniger erklärbar. Diese Jugendlichen befinden sich also in einem starken Abhängigkeitsverhältnis zu den Medien, wenn es um die Vermittlung sozialer Wirklichkeit geht:

„na, eigentlich muss man das doch glauben, oder?... weil, es bleibt einem ja gar nichts anderes

übrig, wir sehen das und na ja, dann wird das schon stimmen..., wie kriegen wir denn raus, dass es nicht stimmt" (Aw; Z. 1024-1032).

In ihren Deutungen unterstellen die Jugendlichen den Medien einen autoritativen Charakter: Welt entzieht sich tendenziell den eigenen Erklärungs- und Deutungsmustern; Sinnkonstruktionen im Verstehen von Welt werden kaum zum Gegenstand selbstreflexiver Auseinandersetzungsprozesse, die Deutung sozialer Wirklichkeit wird so in den Verantwortungsbereich der Medien überführt. Dimensionen medienkritischer Auseinandersetzungen beruhen allein in der Attestierung des Nichteinlösens dieses so formulierten und von den Jugendlichen erwarteten Anspruchs bzw. Auftrages nach medialer Pflichterfüllung. Dort, wo Welt durch die Medien nicht mehr erklärt werden kann, generiert sich Unsicherheit und Nicht-Verstehen und in der Folge Rückzug und Resignation.

3.3 Orientierungs- und Deutungsrahmungen Jugendlicher und ihre Relevanz für Medienkritik - Eine exemplarische Fallrekonstruktion

Diese bis hier dargestellten kollektiven Orientierungsrahmungen im (Medien-) Handeln Jugendlicher formen sich maßgeblich vor dem Hintergrund bildungs- bzw. milieuspezifischer Dispositionen aus. Damit wird auch eine je spezifische Sicht Jugendlicher in der kritischen Auseinandersetzung mit Medien, aber auch mit dem eigenen Medienhandeln generiert, die wiederum eingebettet ist in eine je spezifische Sicht auf eine moderne Welt, in der sich die Jugendlichen auch selbst verorten und positionieren müssen.

Diese komplexen Zusammenhänge sollen in einer nun folgenden exemplarischen Fallrekonstruktion nachgezeichnet werden. Die Interpretation der Gruppendiskussion zeigt, dass das eigene lebensweltlich eingespannte Medienhandeln Jugendlicher in den Kontext gesellschaftlicher Modernisierungs- und Wandlungsprozesse gestellt ist, die wiederum selbst von den Jugendli- 
chen gedeutet, ja ,verstanden` werden (müssen). Medienkritik Jugendlicher - so unsere These - findet ihre fallspezifische Ausprägung in den je konkreten, kommunikativ verhandelten Orientierungsrahmungen Jugendlicher in der Deutung und im Sinnerschließen moderner Welt. Diese spezifischen Sichtweisen Jugendlicher und damit die Sinn- und Bedeutungskonstruktionen im Verstehen und Erklären von Welt generieren Jugendliche auch vor dem Hintergrund medial vermittelter Symbolangebote, die wiederum in Abhängigkeit zum jeweiligen Bildungsniveau bzw. zum sozialen Milieu spezifisch übernommen oder abgelehnt werden. Diese Annahmen legen nun in einem weiteren Schritt der empirischen Untersuchung von Medienkritik - als eine Analyseebene von Medienkompetenz - einen rekonstruktiven Zugang nahe, der eben jene Sinn- und Bedeutungsstrukturen Jugendlicher nachzuzeichnen vermag. In den folgenden Abschnitten steigen wir in Auszüge der Fallrekonstruktion ein.

\subsubsection{Idealisierte Modernisierungsprozesse im historischen Kontext und ihr gegenwärti-} ges Ende-der ,Normalfall' Berufsschule

Wir befinden uns nun - in der Logik eines eher rekonstruktiven Vorgehens - in jenem Teil der Gruppendiskussion, in dem die Jugendlichen ihre Sicht auf die Welt, aber auch ihre milieu- bzw. bildungsspezifisch festgeschriebene Rolle innerhalb dieser Welt kommunikativ aushandeln. Dabei zeigt sich, dass die Spezifik des Falls ganz konkrete Formen des Ausschlusses aber auch der Beteiligung am Prozess der Modernisierung von Welt unterstellt. Innerhalb dieses Prozesses lassen sich die Lebenswelten der Beteiligten einerseits und die Lebenswelten der Nichtbeteiligten andererseits finden, die einen je spezifischen Umgang mit den Unsicherheiten moderner Gesellschaften implizieren. Wir gewinnen hier einen ,Einblick` in die Lebenswelt der Nichtbeteiligten, die vor dem Hintergrund ganz konkreter Erfahrungsdispositionen im Umgang und der Lösung von Unsicherheiten und Risiken im Modernisierungsprozess spezifische kollektive Deutungs- und Orientierungsrahmungen generieren, in die u.E. Medienkritik selbst eingespannt ist, indem sie als Resultante eines spezifischen Erfahrungswissens im Deuten und Verstehen von Welt ,funktioniert'.

Em: Guck mal vor zwanzig Jahren, vor dreißig Jahren

Fm: Das war die Menschheit, die nach Wissen strebte und das ist vorbei, das ist zu Ende die Evolution ist vorbei, das ist Geschichte,

Em: Eh das glaub 'ste nicht

Fm: Jetzt geht's rückwärts, guck doch mal hier rum auf der Schule, guck doch mal dir die Berufsschule aus, wer intelligent ist, und wer ottonormal ist"

(Z. 3262- 3272) ${ }^{4}$

4 Die Transkription der Interviews erfolgte nach einem vereinfachten Verfahren aus Rosenthal (1987). Die Unterstreichung im Text symbolisiert eine Betonung des Sprechers. Die Beteiligten wurden in der Gruppendiskussion alphabetisch zugeordnet (hier: A bis F), die Zuordnung orientiert sich dem ersten Sprachbeitrag. Der zweite Buchstabe (m oder w) kennzeichnet das Geschlecht der Beteiligten. Die angegebenen Zeilen (Z.) sind ein Verweis auf die Zeilen im Interview. 
In der retrospektiven Perspektive auf historische Verläufe (,vor zwanzig, vor dreißig Jahren“) wird der „Menschheit“ das Streben nach Wissen und damit das Streben nach Erkenntnis im Glauben an Fortschritt und Entwicklung unterstellt und gleichsam unter gegenwartsbezogener Perspektive abgesprochen. Damit wird das Ideal von Modernisierung, dessen Gelingen in der Antriebskraft des Erkenntnisgewinns („Wissen“) liegt, in der Gegenwart als nicht eingelöst bzw. als nicht einlösbar gekennzeichnet. Darüber hinaus stehen die Modernisierungsansprüche, also das Streben nach Wissen, konträr zur potenziell möglichen (Weiter-)Entwicklung der Fähigkeiten und Kompetenzen jener Akteure, die diese Modernisierungsansprüche umzusetzen und damit einzulösen haben. Das Verschwinden der ,Idee des Fortschritts' ist somit auch an die Verunmöglichung der Angleichung der Fähigkeiten der „Menschheit“ gebunden, ihre eigenen Modernisierungsansprüche und -erwartungen handlungspraktisch umsetzen bzw. verbürgen zu können (,das ist zu Ende, die Evolution ist vorbei“).

Dies führt zur Fundamentalbilanz, dass die Zeit der Neuerung, des Fortschritts, der (Weiter-)Entwicklung und damit auch der Modernisierung als abgeschlossen gilt und allenfalls im historischen Kontext zu verorten ist („das ist Geschichte“). Argumentativ wird diese Aburteilung der Gesellschaft in der Ausdifferenzierung von Bildungsniveaus und sozialen Milieus festgemacht, die sich hier exemplarisch in der Miniaturfigur „Berufsschule“ manifestieren. Denn, so wird unterstellt, dass es vor „zwanzig Jahren, vor dreißig Jahren" noch eine, in gewissem Sinne homogene Gesellschaftsstruktur (,Menschheit“) gegeben hat, für die das Streben nach Wissen bezeichnend war. Dieser Zeit wird unter der Hand einerseits ein spezifischer Bildungsbegriff (,Wissen“) und andererseits ein spezifischer Bildungsanspruch (,,Streben nach Wissen“) unterstellt.

So kann das ,Streben nach Wissen“ hier als homogener Bildungsanspruch gedeutet werden, der den historisch-epochalen Drang nach Bildung und damit nach Erklärbarkeit, Erkenntnis und Verstehen von Welt unterstellt. Diese so hergestellte Geschlossenheit löst sich in der Gegenwart insofern als auf, als sich in ihr Bildungsansprüche und somit auch Bildungsniveaus kontrastiv entwickeln (,intelligent“; „ottonormal“). Die Berufsschüler stilisieren sich in diesem Zusammenhang tendenziell als Repräsentanten eines spezifischen Bildungsniveaus („ottonormal“), die nicht als Garanten der Umsetzung bzw. Einlösung von Modernisierungsansprüchen bzw. -erwartungen auftreten.

Modernisierungsprozesse sind in ihrer Einlösung bzw. Umsetzung somit strikt an ihre professionellen Repräsentanten gebunden. Sinnlogisch sind es demnach die „,intelligent(en)“,Eliten', die überhaupt an Modernisierungsprozessen partizipieren und erst so das idealisierte ,Vorwärts‘, also die gesellschaftliche Entwicklung, aber auch die Entwicklung ihres eigenen Selbst 
(,Evolution“), nicht zuletzt vor dem Hintergrund adäquater Bildungsniveaus (,intelligent"), entsprechend einlösen. Vor dem Hintergrund des dazu konkurrierenden milieubzw. bildungsspezifischen Kontextes „Berufsschule“, diagnostizieren die Jugendlichen Gegenläufiges. Denn so ist es in Abgrenzung zu den ,intelligenten Eliten' der gesellschaftliche ,Durchschnitt“ (,ottonormal“), der entgegen der ,Vorwärtsgewandtheit“ von Modernisierungsprozessen eher ,Rückwärtsgewandtheit', zumindest jedoch Stagnation im Sinne des Nicht-Einlösens bzw. Nicht-Einlösen-Könnens entsprechender Modernisierungsanforderungen symbolisiert.

Zweifel an diesem Entwurf (,eh das glaub 'ste nich“) können durch eine evaluative ,Aufrechnung' eben jener Akteure, die über die spezifischen Modernisierungsbefähigungen verfügen (,intelligent“), und denjenigen Akteuren, die vor dem Hintergrund ihrer Fähigkeiten Modernisierung eher verhindern (,ottonormal“), ausgeräumt werden. Dabei fällt auf, dass in dieser Kontrastierung von „intelligent“ und „ottonormal“ zwar Statusunterschiede in den Bildungsniveaus markiert werden, die jedoch nicht zwangsläufig eine gleichzeitige Entwertung der ,rangniederen“ Gruppe der „Ottonormale(n)“ nach sich zieht. Denn so sind es gerade diejenigen („ottonormal“), die vor dem Hintergrund so implizit thematisierter Normalitätsentwürfe der ,Normalität‘ entsprechen. Die Gruppe der „Intelligenten“ wird somit im Kontext des hier spezifischen Bildungsniveaus („Berufsschule“) zur nicht-repräsentierten Ausnahme, die dem Normalfall entgegenläuft. Die Gültigkeit dieses (Normalitäts-)Entwurfs kann jederzeit durch jeden ,überprüft‘ werden, der sich legitim als ,Insider‘ oder ,Kenner` dieses konkreten Kontextes (,auf der Schule“) ausweisen kann und der sich vor dem Hintergrund seiner analytischen Fähigkeiten („guck doch mal hier rum“) eben jenen repräsentativen Normalitätsentwurf erschließt. Der konkrete institutionelle Zusammenhang Berufsschule wird so zum repräsentativen, aber auch zum identitätsstiftenden Moment der hier vertretenen Akteure. Die Perspektive eines Berufsschülers repräsentiert in dieser Logik also eine spezifische Sichtweise, nämlich eine aus dem Selbstverständnis als ,normalisierter Durchschnitt‘. So wird dieser stellvertretenden Deutung übergreifende Gültigkeit unterstellt, die gemeinhin für den berufsschulspezifischen Kontext bindend ist. Diese stellvertretende Deutung problematisiert gleichzeitig in der Gegeneinanderführung historischer und gegenwärtiger Bezüge den Verlust gesellschaftlich homogener Bildungsansprüche durch die Säkularisierung in spezifische Bildungsniveaus.

Dabei ist entscheidend, dass der im historischen Kontext verortete Bildungsanspruch dem Idealbild von Fortschritt und Entwicklung - also von Modernisierung - entspricht, denn nur in diesem Idealbild der übergreifend kollektiv verbürgten Idee des Erklärens und Verstehens von Welt erscheint Vorwärtsgewandtheit, also (Weiter-)Entwicklung und Fortschritt garantiert. Gegenwärtig jedoch ist bezüglich dieser Idealkonstruktion eine historische Zäsur eingeführt, die kollektive Verbürgung nicht mehr garantiert. In der Deutung der Jugendlichen wird vielmehr die Gesellschaft in ,Beteiligte (aktive, die Entwicklung der Moderne verstehende und steuernde Personen) und 
,Nicht-Beteiligte‘ (passive, von den Entwicklungen der Moderne lediglich betroffene und getriebene Personen) aufgeteilt. Kollektive Verbürgung garantierende Bildungsansprüche haben sich in voneinander unterscheidbare, institutionalisierte Bildungsniveaus verkehrt, die ihre je spezifischen Möglichkeiten bezüglich der Partizipation an Modernisierung beinhalten bzw. festlegen. Der hier ausgewiesene berufsschulspezifische Kontext steht in der Tendenz als Repräsentation einer - im Selbstverständnis der jugendlichen Akteure - institutionell organisierten Gemeinschaft der ,Nicht-Beteiligten`.

Bis hier bleibt Folgendes festzuhalten: Im stellvertretend gedeuteten Selbstbild der Jugendlichen als Berufsschüler werden bildungsspezifische Unterscheidungen aufgemacht, die jeweilige Formen der Beteiligung am Modernisierungsgeschehen nahe legen oder verschließen. Die Möglichkeit solcher milieu- bzw. bildungsspezifischen Unterscheidungen werden dem gegenwartsbezogenen Kontext unterstellt und sind somit als Ausdruck einer modernen Gesellschaft zu sehen, die sich nicht nur von ihrem Idealbild von Modernisierung als kollektiv geteilte Idee des Fortschritts und der Weiterentwicklung entfernt, sondern tendenziell soziale Ungleichheit produziert. So sehen sich hier die benachteiligten Berufsschüler in die Position der Nichtbeteiligten gedrängt, die den ,intelligenten Modernisierungseliten` gegenüberstehen. Diese Ausgrenzung wird von den Berufsschülern in der Verteidigung ihrer sozialen Rolle als ,normaler Durchschnitt‘ beantwortet. In dieser Selbstpositionierung der Berufsschüler werden somit milieu- bzw. bildungsspezifische Unterscheidungen konstruiert, die ausschlaggebend dafür sein können, welche je spezifischen (medien-)kritischen Bezüge Jugendliche ausformen und welche vor dem Hintergrund ihrer Ausgrenzungserfahrungen per se ausgeschlossen sind. So wurden bis hier Erfahrungsdispositionen Jugendlicher nachgezeichnet, die eher Verweigerung bzw. Rückzug betonen, als dass sie eine aktive Teilhabe dieser Akteure in der reflexiv-kritischen und gestalterischen Auseinandersetzung innerhalb gesellschaftlicher Modernisierungsprozesse ausformen.

Im nächsten Abschnitt wird nun der Frage nachgegangen, welchem Adressaten das Gelingen des Modernisierungsprojekts überantwortet wird.

\subsection{2 Über den Verlust charismatischer Repräsentanten und die Verbürgung von Moder-} nisierung

„ich muss schon sagen, dass, dass och in damaligen Zeiten irgendwie irgendwas anderes im Vordergrund stand, zum Beispiel die Erfinder, zum Beispiel Einstein, der war weltberühmt, wenn man jetzt irgendeinen Erfinder der irgendeine Formel erfunden hat, da weeß keener den Namen“ (Em; Z. 2840- 2846).

Einmal mehr scheint sich der retrospektive Blick in die „damalige Zeit“ zu lohnen. Dass sich diese Zeit im Vergleich zum gegenwartsbezogenen Kontext durch eine Besonderheit auszeichnet, wird zwar betont (,im Vordergrund stand“), jedoch nicht weiter spezifiziert (,irgendwie irgendwas“). So werden hier zunächst einmal nur Differenzen zwischen historischem und ge- 
genwartsbezogenem Kontext markiert. Diese Differenzen bzw. Prioritäten (,im Vordergrund stand“) werden im Folgenden an einem „Beispiel“ erklärt. So sind es die „Erfinder“ als personalisierte Repräsentanten des Fortschritts und der Entwicklung „damaliger Zeiten“ - im Speziellen ist es „Einstein“ als ,nennenswerter“ Repräsentant einer Profession („Erfinder“) - und dieser so bedeuteten Zeit des Glaubens an Entwicklung und Fortschritt. In diesem Sinne müssen die ,alten Zeiten` mit Blick auf die Vergangenheit als Garant für Entwicklung und Fortschritt herhalten. Die personalisierten Repräsentanten stehen also für die Idee der Modernisierung überhaupt. Darüber hinaus verkörpert diese Personalisierung, die nicht zuletzt „Einstein“ „weltberühmt“ gemacht hat, eher eine nicht genau benennbare Vergangenheit und verliert sich unter den gegenwärtigen Bezügen in der X-Beliebigkeit. So sind es heute (,jetzt“) „irgendwelche“, scheinbar bedeutungslos gewordene Formeln von irgendwelchen „Erfinder(n)“, die die Idee von Modernisierung kaum mehr verbürgen. Zwar sind auch in der heutigen Zeit Modernisierungsaspirationen, die durch „Erfinder“ und „Formeln“ immer wieder neu angestoßen werden, nicht obsolet geworden. Jedoch werden sie in der Gegeneinanderführung zur „damaligen Zeit“" nicht durch charismatische Repräsentanten einer Profession in der Weise verstetigt, wie sie erst dadurch übergreifende Gültigkeits- und Bewährungsansprüche (,weltberühmt“) erheben können.

In diesem Bilde stehen „Erfindungen“ und „Formeln“ für sinngenerierende ,neue Technologien', derer man sich bedient, um die Welt erklären und gestalten können. So sind es aber auch jene Technologien, die überhaupt erst die Machbarkeit des Verstehens, Erklärens und Gestaltens von Welt unterstellen, ohne jeden Zweifel, trotz permanenter, eigendynamisch verlaufender Wandlungs- und Veränderungsprozesse. Das Gelingen hängt dabei einerseits immer davon ab, wie gut eben jene Neuerungen selbst in der Lage sind, (neuen) handlungsleitenden Sinn, also Wirklichkeit, Orientierungen und Sicherheiten zu generieren und zu versprechen. Andererseits - und das zeigt dieser Fall - können sie dies immer weniger aus sich selbst heraus. Denn das Gelingen bzw. die Machbarkeit des Erklärens und Gestaltens von Welt, das immer auch gebunden ist an das Streben um das ,richtige` Wissen, hängt ab von der kollektiven Verbürgung. Der eigentliche Erfolg von Modernisierung liegt demzufolge in seinem gelungenen, (kollektiv) glaubhaften Versprechen. Dies gilt hier nur dann als gelungen, wenn Geltungs- und Bewährungsansprüche eben dieser ,Neuerungen` umfassende Gültigkeit und Anerkennung (,weltberühmt“) erfahren und wenn diese in einer konkret wahrnehmbaren, personellen Repräsentanz verschmelzen. So empfinden hier die Akteure Modernisierungsprozesse nur dann als adäquat, wenn sich die Versprechen der Moderne handlungspraktisch auch nachvollziehen lassen, wenn sie also gemeinhin das einlösen, was sie verheißen, nämlich Erklärbarkeit, Verstehen und Nachvollzug von Fortschritt und Entwicklung. Dies wird jedoch in diesem Fall der „damaligen Zeit“ zugeschrieben, wobei es in der Gegenwart zwar solche (Weiter-)Entwicklungen immer noch gibt, jedoch verweisen diese kaum noch auf eine übergreifende Verbürgung in der Etablierung der Idee 
des Fortschritts und der Entwicklung. Modernisierungsprozesse verlieren also in der Gegenwart für die Jugendlichen in der Anonymisierung einer modernisierenden Elite (,irgendeinen Erfinder") ihre verbürgte und übergreifende Idee an Fortschritt, Entwicklung und Modernität. Modernisierung bleibt für sie allenfalls noch als permanenter Wandel erfahrbar.

War es somit im historischen Kontext also das kollektive Streben nach Wissen und damit nach Erklärbarkeit und Verstehen von Welt, so ist es unter gegenwärtigen Bezügen der dramatische Verlust von Wissen im Nachvollzug um die ,eindeutig richtigen` Technologien - im Sinne sinngenerierender Handlungsalternativen. Damit generiert sich vor dem Hintergrund unterschiedlicher Bildungsniveaus eine Wissenskluft im Nachvollzug und dem Verstehen von Modernisierung, die sich im konkreten Fall vor dem Hintergrund des berufsschulspezifischen Kontextes repräsentiert.

\subsubsection{Modernisierungsprozesse versus Modernisierungsschub - wann verliert Moderni- sierung ihren Sinn? \\ „aber ich muss schon sagen, dass die Entwicklung ziemlich schnell geht, dass mich das ziemlich erschreckt“ (Em; Z. 2983- 2985).}

Modernisierungsprozesse werden vor diesem Hintergrund als relativ eigendynamische, sich selbst reproduzierende Abläufe charakterisiert („Entwicklung...geht“), die in ihrem Verlauf und ihrer Geschwindigkeit (,ziemlich schnell“) nicht nur Distanzen und das Gefühl von Angst und Unbehagen produzieren („erschreckt“). Modernisierung erscheint immer weniger als prozessual generiert, was den Akteuren die Möglichkeit verschließt, an diesem Prozess der Generierung ,neuen Wissens“ und ,neuer Technologien“ („Ideen“) partizipieren zu können. Vielmehr steht Modernisierung hier für einen unkontrollierten, Distanz erzeugenden und Partizipation verunmöglichenden Modernisierungsschub, der freilich auch das Gefühl der Lähmung und der Angst erzeugt. Was bleibt, ist die Wahrnehmung von Modernisierung als gewaltiger Modernisierungsschub, der eher in der Figur des permanenten Wandels, im Sinne ebenso permanenter Re-Formulierungen von ,neualten' Sicherheiten und Orientierungen erscheint, statt der Wahrnehmung von Modernisierung als der gelungenen Generierung und Umsetzung ,neuer Technologien', um den Anforderungen der Moderne erkenntnis-, sinn- und orientierungsstiftend gerecht werden zu können. Modernisierung verfehlt demzufolge in der Idealkonstruktion ihr Ziel und erreicht genau das Gegenteil: Desorientierung durch den Verlust von Sicherheiten und gemeinsam getragenen Ideen, verbunden mit der tendenziellen Ausgrenzung der - hier repräsentierten - Akteure aus dem Modernisierungsgeschehen.

„für mich einfach viel zu schnell, man schnallt das eigentlich gar nicht alles=alles, was jetzt (...) erfindet, erfunden wurde“ (Em; Z. 2996-2999) 
Die Konsequenz eben jener Modernisierungsschübe liegt somit auf der Hand. Indem Modernisierungen als „einfach viel zu schnell“ wahrgenommen werden, bleibt nicht nur der Nachvollzug und das Verstehen jener Entwicklungen aus („man schnallt das eigentlich gar nicht alles"). Vielmehr verschließt sich auch Partizipation an diesen Entwicklungen. Hier verfehlt - genau genommen - die bereits angedeutete Wissenskluft ihre Wirkung nicht: Das fehlende und dennoch so wichtige Wissen im Modernisierungsgeschehen generiert ebenso die Verunmöglichung des Nachvollzuges und des Verstehens von Modernisierung. Dies impliziert die fatale Folge dieser so charakterisierten Modernisierungsverläufe: ,Erfindungen` können - wie bereits angedeutet - als der Schlüssel zur Generierung ,neuen Wissens‘ auch im Sinne alternativer Handlungsoptionen bedeutet werden, über die wiederum Modernisierung überhaupt erst erklärbar, verstehbar und somit nachvollziehbar wird. Verstehen sich diesbezüglich die konkreten Akteure als Ausgeschlossene, die sich diesen Entwicklungen im Verstehen und Erkennen nicht angleichen können, verschließt sich damit auch der Sinnbezug von Modernisierung und damit auch der Sinnbezug im Erschließen des ,Neuen‘. Modernisierung, Fortschritt, Entwicklung - all das sind komplexe Prozesse, die ebenso in unser Leben und unsere Gewohnheiten bzw. Alltagsroutinen eingreifen, wie sie damit eine Steigerung der Lebensqualität bzw. unseres Lebensstandards verheißen. Nun verkommen aber in der Gegenwart gerade jene ,Modernisierungseliten` zum Gegenteil, nämlich zu namenlosen, anonymen Modernisierungsbeteiligten. Die immer schnelleren und eigendynamisch verlaufenden Modernisierungsverläufe, die den Nachvollzug und das Erfahren und Verstehen der so quasi schon unterstellten Neuerungen für die Nichtbeteiligten tendenziell verunmöglichen, führen in der Folge nicht nur zu einem Ausschluss dieser Akteure in der ,Generierung des Neuen‘. Vielmehr scheint Modernisierung (als Idee) strukturell ihren orientierungsstiftenden Sinn zu verlieren.

Bis zu dieser Stelle werden in den Textpassagen zwei konkurrierende Entwürfe von Modernisierung mit ihren jeweiligen Konsequenzen für die konkreten Akteure präsentiert: Einmal wird in der retrospektiven Perspektive auf ein Idealbild von Modernisierung Bezug genommen, dem das Streben nach ,neuem Wissen', also gemeinhin das Streben nach Fortschritt und Entwicklung, unterstellt wird. Diese so formulierten Modernisierungsideale gelten im historischen Kontext in ihren Geltungs- und Bewährungsansprüchen als umfassend eingelöst und anerkannt. Dies nicht zuletzt durch die personelle Verbürgung und Einlösung dieser so formulierten Ideale durch herausgehobene modernisierende Repräsentanten einer historisch bereits abgeschlossenen ,Blütezeit‘ Modernisierungsprozesse im Rahmen dieses historischen Kontextes gehen im Bild der personell verbürgten Modernisierung als ,Idee des Fortschritts und der Entwicklung‘ auf.

Diesen Modernisierungsprozessen der ,damaligen Zeit‘ stehen Modernisierungsverläufe in der Gegenwart gegenüber, die weitestgehend willkürlich und eigendynamisch verlaufen und eher den Charakter distanzerzeugender Modernisierungsschübe aufweisen. Dies führt in der Konsequenz zum ,pas- 
siven Erleiden` gesellschaftlicher Wandlungsprozesse, die vor dem Hintergrund der formulierten Idealbilder von Modernisierung kaum noch einen Nachvollzug bzw. eine Einordnung sinngenerierender Modernisierungsaspirationen zulassen und in der Tendenz eher Desorientierungen und Verunsicherungen provozieren. Vielmehr jedoch generieren bzw. potenzieren diese Modernisierungsverläufe Wissensklüfte im Nachvollzug und Verstehen in Abhängigkeit zu den konkret repräsentierten Bildungsniveaus.

\section{Schlussfolgerungen}

Führt man nun die aus diesen beiden hier vorgestellten Zugängen in der empirischen Erschließung von Medienkompetenz generierten Ergebnisse zusammen, so lassen sich folgende erkenntnisgenerierenden Aussagen dieses exemplarischen Falles festhalten:

Das Medienhandeln der befragten Jugendlichen ist eingebettet in ihre konkreten Weltdeutungen, die in spezifischer Art und Weise und vor dem Hintergrund milieu- und bildungsspezifischer Dispositionen wahrgenommene Phänomene (hier die Medienberichterstattung des 11. September 2001) zu verstehen und einzuordnen suchen.

In Auseinandersetzung mit außergewöhnlichen oder irritierenden (Medien-)Ereignissen, die als Handlungskrisen bezeichnet werden können, greifen die Jugendlichen also auf ihr vorhandenes Erfahrungs- und Deutungswissen zurück. Das reflexive Wissen Jugendlicher um die Abläufe und Strukturlogiken eines Mediensystems, das in gewisser Weise die Paradoxien moderner Gesellschaften selbst reproduziert, speist sich also aus den lebensweltlich generierten Orientierungs- und Deutungsrahmungen und bedingt gleichsam die fallspezifische Ausprägung von Fähigkeiten im kritischen Umgang mit Medien. Medienkritik kann demzufolge nicht per se als erwartbare Festlegung an den Fall herangetragen werden, sondern muss sich aus dem Fall selbst, also vor dem Hintergrund der spezifisch generierten kollektiven Orientierungs- und Deutungsrahmungen Jugendlicher herleiten lassen. Erst so kann u.E. erklärt werden, welche Besonderheiten in den Lebenswelten Jugendlicher dafür ausschlaggebend sein können, dass sich im Medienhandeln Jugendlicher kaum Medienkritik zeigt.

Um es noch einmal zu wiederholen: Die ,medien-kritische' Position der von uns befragten Jugendlichen beschränkte sich im Wesentlichen auf das Bedauern, dass seinerzeit die Medien zwar permanent über den Anschlag auf das WTC mit Wort und Bild berichteten, aber keine eindeutigen Bewertungen und Gründe dafür lieferten. Die Jugendlichen hätten sich eine ,klare Berichterstattung' gewünscht, durch die dann der Vorfall aber auch medial ,erledigt‘, gewissermaßen ,abgehakt‘ worden wäre. Aber statt dieser gewünschten eindeutigen Berichterstattung der Medien mussten die Jugendlichen nicht nur divergierende Berichte, Vermutungen und konträre Meinungen aushalten. 
Die Medienlandschaft (vor allem das Fernsehen) ließ den eigentlich unterhaltungsorientierten Jugendlichen keine Nischen des ,Entkommens' mehr: Überall und andauernd wurde nur noch über den Anschlag berichtet. In der Weltdeutung der Jugendlichen konnten sie als „Ottonormale“ diese uneindeutige Dauerberichterstattung der Medien nicht angemessen einschätzen. Was genau geschehen war, wer schuldig, Täter oder Drahtzieher war, entzog sich ihrer Kenntnismöglichkeit. Wie bei anderen komplizierten Phänomenen (z.B. neuere Physik oder allgemein: die komplexen Vorgänge und Interdependenzen der Moderne) lässt es in der Selbstsicht der Jugendlichen ihr Ausbildungsstand (eigentlich ihr gesamtes biografisches Schicksal als „Ottonormale“) nicht zu, Hintergründe und Durchblicke angemessen selbst zu analysieren. Für sie bleiben nur die Möglichkeiten an Kritik: unverstehbare Dinge zu personalisieren (wie z.B. neuere Physik in der Person „Einstein“, der man früher noch vertrauen/nicht vertrauen oder der man glauben/ nicht glauben konnte), oder eben von den Medien eindeutige Bilder und Fakten geliefert zu bekommen, denen man genauso vertrauen/nicht vertrauen oder der man glauben/nicht glauben kann.

Eben dieser ,Serviceleistung ‘ aber hatten sich die Medien in der medienkritischen Sicht der Jugendlichen rund um den 11. September entzogen. Statt Klarheit in den politisch komplexen und undurchschaubaren Vorfall zu bringen, wurde er zum medialen Dauerthema perpetuiert. Die befragten Jugendlichen als „Ottonormale“ reagierten darauf wie auf andere Unerklärbarkeiten und Irritationen der Moderne auch. Ihre kritische Haltung dazu aktualisierte ein auch in anderen Kontexten bewährtes Deutungsmuster, nach dem die „Ottonormalen“ nicht nur die Katastrophen und Imponderabilitäten der Moderne aushalten und erleiden müssen, sondern zusätzlich von den Eliten (hier den Medienmachern) auch noch unaufgeklärt bleiben. Zusätzlich gewährten die Medien um den 11. September den „Ottonormalen“ dann noch nicht einmal das, was ihnen überhaupt noch zu tun übrig blieb; nämlich den Leerraum der eigenen Eingreif- und Mitgestaltungsmöglichkeiten durch den Genuss von Spielfilmen und anderer Medienunterhaltung zu füllen.

\section{Literatur}

Baacke, D.: Kommunikation und Kompetenz. Grundlegung einer Didaktik der Kommunikation und ihrer Medien. München 1980 (3. Aufl., Orig. 1973).

Baacke, D.: Medienkompetenz - Begrifflichkeit und sozialer Wandel. In: Rein, A. v. (Hrsg.): Medienkompetenz als Schlüsselbegriff. Bad Heilbrunn 1996, S. 112-124.

Baacke, D.: Medienpädagogik. Tübingen 1997.

Baacke, D./Sander, U./Vollbrecht, R. u.a.: Zielgruppe Kind. Kindliche Lebenswelten und Werbeinszenierungen. Opladen 1999.

Barthelmes, J./Sander, E.: Medien in Familie und Peer-group. München 1997. 
Bohnsack, R.: Gruppendiskussionsverfahren und Milieuforschung. In Friebertshäuser, B./Prengel, A. (Hrsg.): Handbuch qualitative Forschungsmethoden in der Erziehungswissenschaft. Weinheim 1997, S. 492-502.

Bourdieu, P.: Über das Fernsehen. Frankfurt a.M. 1998, S. 21.

Dewe, B./Sander, U.: Medienkompetenz und Erwachsenenbildung. In: von Rein, A. (Hg.): Medienkompetenz als Schlüsselbegriff. Bad Heilbrunn 1996, S. 125-142. Fromme, J./Kommer, S./Mansel, J./Treumann, K. P. (Hrsg.): Selbstsozialisation, Kinderkultur und Mediennutzung, Opladen 1999.

Loos, P./Schäffer, B.: Das Gruppendiskussionsverfahren. Opladen 2001.

Oevermann, U.: Zur Analyse der Struktur von sozialen Deutungsmustern (Original: 1973). In : Sozialer Sinn. Zeitschrift für hermeneutische Sozialforschung, H.1, 2001, S. 3-11.

Oevermann, U.: Die Struktur sozialer Deutungsmuster - Versuch einer Aktualisierung. In: Sozialer Sinn. Zeitschrift für hermeneutische Sozialforschung. H. 1, 2001a, S. 35-83.

Rosenthal, G.: „...wenn alles in Scherben fällt...“. Vom Leben und Sinnwelt der Kriegsgeneration. Typen biographischer Wandlungen. Opladen 1987

Thole, W.: Jugend, Freizeit, Medien und Kultur. In: Krüger, H.-H./Grunert, C. (Hrsg.): Handbuch Kindheits- und Jugendforschung. Opladen 2002; S. 653- 685.

Treumann, K. P.: Tringulation als Kombination qualitativer und quantitativer Forschung: In: Abel, J./Möller, R./Treumann, K. P. (Hrsg.): Einführung in die Empirische Forschung. Stuttgart 1998, S. 154-182.

Treumann, K. P./Baacke, D./Haacke, K./Hugger, K.-U./Vollbrecht, R.: Medienkompetenz im digitalen Zeitalter. Wie die neuen Medien das Leben und Lernen Erwachsener verändern. Opladen 2002. 